\title{
Adult cardiac veno-arterial extracorporeal life support (VA-ECMO): prevention and management of acute complications
}

\author{
Michael M. Koerner, Michael D. Harper, Christopher K. Gordon, Douglas Horstmanshof, \\ James W. Long, Michael J. Sasevich, James D. Neel, Aly El Banayosy \\ Advanced Cardiac Care and Transplant Institute, Integris Baptist Medical Center, Oklahoma City, OK, USA \\ Correspondence to: Aly El Banayosy, MD. Executive Director Critical Care, Director Acute Circulatory Support, Advanced Cardiac Care/Transplant Institute, \\ Integris Baptist Medical Center, 3400 NW Expressway, Bldg C, Suite 300, Oklahoma City, OK 73112, USA. Email: Aly.ElBanayosy@integrisok.com.
}

\begin{abstract}
Background: Veno-arterial extracorporeal membrane oxygenation (VA-ECMO) has been shown to be a viable and effective treatment for patients suffering from refractory cardiogenic shock (rCS), which is associated with high mortality rates. Although ECMO therapy used as short-term mechanical circulatory support (MCS) has shown tremendous growth in its application over the past decade, the complication and mortality rates remain high. This retrospective study analyzes complications associated with VA-ECMO support, evaluates the use of defined protocols at a single center, and examines factors that may contribute to patient complication and mortality.

Methods: This retrospective analysis included 184 patients who were supported with ECMO from September 2014 through March 2018 at Integris Baptist Medical Center (IBMC). Descriptive statistics were generated to analyze baseline characteristics, demographics, complications, and outcomes.

Results: Acute myocardial infarct (AMI) was the primary etiology of this cohort ( $\mathrm{N}=40 ; 22 \%)$. The mean age was 55 \pm 15 (median 56, range 15-84) years. All patients were inotrope and/or vasopressor dependent prior to ECMO initiation. Mean time on ECMO support was 7.8 7.9 days with median time of 6 days. Total patient days on support were 1,430. Most ECMO cannulations, 97 (52\%) were performed within Integris Baptist Medical Center, with 48\% done outside the hospital; 38\% were performed outside of the hospital by the IBMC ECMO team, and $10.5 \%$ were performed by an outside team. Bleeding was noted to be the most common VA ECMO complication [ $\mathrm{N}=41 ; 22.3 \%$; 0.028 events per patient day (EPPD)].

Conclusions: A dedicated 24/7 ECMO service using a multidisciplinary team (MDT) and defined protocols in a single center is able to effectively reduce complications due to VA-ECMO support in the sickest of the sick VA-ECMO patients.
\end{abstract}

Keywords: Veno-arterial extracorporeal membrane oxygenation (VA-ECMO); ECMO complications; cardiogenic shock

Submitted Dec 06, 2018. Accepted for publication Dec 20, 2018.

doi: 10.21037/acs.2018.12.09

View this article at: http://dx.doi.org/10.21037/acs.2018.12.09

\section{Introduction}

Despite conventional therapies, refractory cardiogenic shock (rCS) continues to be a life-threatening condition with poor prognosis and unacceptably high complication and mortality rates. Left untreated, derangements within the circulatory system create a low cardiac output state and a resultant cascade of symptoms; systemic hypoperfusion, end organ failure, systemic inflammatory response, and eventually death (1-3). Even with standard interventions, therapies, and medical management, 1-year mortality rates remain high at almost $50 \%(3-5)$.

Although veno-arterial extracorporeal membrane oxygenation (VA-ECMO) therapy has seen a rapid increase in use worldwide, there is no overarching consensus for patient management once on device. An experienced 


\begin{tabular}{|c|c|}
\hline ECMO device related & Patient related \\
\hline Circuit: incorrect connections; clamped tubing & Bleeding: insertion site \\
\hline Oxygenator issues: air embolism, hemolysis & Thromboembolic \\
\hline Pump: leak & HIT \\
\hline $\begin{array}{l}\text { Cannulation related: venous cannula to high (SVC) or below diaphragm; } \\
\text { cannula size too small-reduced flow }\end{array}$ & Vascular: dissection, atherosclerosis, pre-existing filters \\
\hline Oxygen blender: flow too high or too low; not connected to oxygen tank & $\begin{array}{l}\text { Ischemia of lower extremities } \\
\text { Neurologic } \\
\text { SIRS } \\
\text { Significant infection (locally, systemically) } \\
\text { Hemodynamic complications (valve disorder, volume status) } \\
\text { LV dilatation with pulmonary edema; non-contractile LV }\end{array}$ \\
\hline
\end{tabular}

VA-ECMO, veno-arterial extracorporeal membrane oxygenation; HIT, heparin induced thrombocytopenia; SIRS, systemic inflammatory response syndrome.

multidisciplinary team (MDT) is crucial when supporting a successful ECMO program. A risk/benefit analysis should be performed to help to establish guidelines for the appropriate application of VA-ECMO for rCS patients, as not all are the same, and shock states differ in severity and clinical appearance. Understanding the limitations and risks for the application of VA-ECMO is essential, as complication and mortality rates continue to be challenging for this acute therapy.

The underlying disease and duration of VA-ECMO support place already critically-ill patients at risk for developing subsequent serious complications (6). Complications can be classified as either patient related or device related and typically fall under the following categories: (I) bleeding and coagulation; (II) vascular; (III) neurologic; (IV) lower extremity ischemia; (V) systemic inflammation and significant infection; (VI) hemodynamic complications and LV distention; (VII) cannulation complications; and (VIII) device malfunction/failure (Table 1).

The purpose of this study was to characterize the incidence and type of complications for patients undergoing VA-ECMO support and to evaluate effectiveness and impact of (I) clinical practice guidelines; and (II) the MDT for improved outcomes in this cohort of patients at a single center.

\section{Methods}

Data was collected for 184 adults receiving VA-ECMO at a single center between September 2014 and March 2018. A retrospective analysis was performed to assess complication rates, outcomes, and survival in this cohort. Baseline demographics, laboratory values, rCS etiology, VA-ECMO application characteristics and outcomes were analyzed. Freedom from death/mortality was described as patients still alive after the initiation of VA-ECMO support at time of discharge from the hospital. Primary complications were identified and quantified based on severity and incidence.

\section{Statistical analysis}

IBM SPSS Statistics version 22 (IBM Corporation, Armonk, NY, USA) was used to compare and calculate statistical measures for the data. Descriptive statistics were used for categorical data and continuous data was summarized using median (range: minimum to maximum) and mean ( \pm standard deviation) values. Where appropriate, complication rates are reported as events per patient day (EPPD). 


\begin{tabular}{|ll}
\hline \multicolumn{2}{l}{ Table 2 Etiology of cardiogenic shock $(\mathrm{n}=184)$} \\
\hline Variable & $\mathrm{N}(\%)$ \\
\hline AMI shock & $40(21.7)$ \\
\hline Post-cardiotomy shock & $38(20.7)$ \\
\hline Acute on chronic & $26(14.1)$ \\
\hline IHCA & $27(14.6)$ \\
\hline Severe septic shock/SIRS & $18(9.8)$ \\
\hline Myocarditis & $5(2.7)$ \\
\hline Post cardiac transplant & $7(3.8)$ \\
\hline PE & $8(4.3)$ \\
\hline Miscellaneous (hypothermia, pulmonary & $11(6.0)$ \\
\hline hypertension, hypoxia, LVAD failure, poly & \\
\hline trauma) & \\
\hline
\end{tabular}

AMI, acute myocardial infarction; IHCA, in-hospital cardiac arrest; SIRS, systemic inflammatory response syndrome; PE, pulmonary embolus; LVAD, left ventricular assist device.

\section{Results}

\section{Patient characteristics}

During the study timeframe, there were 184 patients who received VA-ECMO for $\mathrm{rCS}$ (Table 2). Their age range was $15-84$ (mean $55 \pm 15$ ) years, with the majority being male $(71 \%)$. The most common etiology of rCS was acute myocardial infarction (AMI), $21.7 \%$, followed by $20.7 \%$ post-cardiotomy rCS. All patients were on a minimum of 2 inotropic agents at the time of VA-ECMO application and $89.6 \%$ were additionally on mechanical ventilation; $31.4 \%$ of those who required acute rCS support were on another type of temporary mechanical circulatory support (MCS) at time of ECMO initiation; $16 \%$ of the 184 patients required continuous renal replacement therapy (CRRT) for acute kidney injury; $46.7 \%$ of patients were transferred from an outside hospital to our ECMO Specialty Center already on VA-ECMO support (Table 3).

In the 184 patients, bleeding (systemic, retroperitoneal hematoma, tamponade) occurred in $\mathrm{n}=41(22.3 \%)$ : cannularelated in $6.0 \%(\mathrm{n}=11)$ and GI bleed in $7.6 \%(\mathrm{n}=14)$. Device related complications (circuit, oxygenator) occurred in $16.8 \%(\mathrm{n}=31$, and $0.002 \mathrm{EPPD})$ of patients: oxygenator failure $3.3 \%(n=6)$, oxygenator clots $4.3 \%(n=8)$. Hemolysis was observed in $9.2 \%(\mathrm{n}=17)$, and ECMO-related neurologic injury in $5.4 \%$ ( $\mathrm{n}=10 ; 0.006 \mathrm{EPPD})$. Non-ECMO related neurologic injury occurred in $6.5 \% \quad(n=12)$. Pulmonary

\begin{tabular}{|c|c|}
\hline Variables & Outcomes \\
\hline Age (years) & $55 \pm 15[15-84]$ \\
\hline Female gender (\%) & $53(29 \%)$ \\
\hline BSA & $2.1 \pm 0.3(1.4-3.0)$ \\
\hline Inotropic/pressor-dependent & $180(98 \%)$ \\
\hline MAP $(\mathrm{mmHg})$ & $60 \pm 15$ \\
\hline IABP & $41(22 \%)$ \\
\hline Pre-ECMO Impella & $12(7 \%)$ \\
\hline CRRT & $29(16 \%)$ \\
\hline SOFA score & $13 \pm 3$ \\
\hline Lactic acid (mmol/L) & $7.4 \pm 3.8$ \\
\hline Platelets & $165 \pm 93$ \\
\hline WBC & $14 \pm 8$ \\
\hline Bilirubin (mg/dL) & $1.9 \pm 2.0$ \\
\hline Creatinine (mg/dL) & $2.1 \pm 1.6$ \\
\hline BUN & $33 \pm 19$ \\
\hline AST & $590 \pm 1,312$ (median 103) \\
\hline \multicolumn{2}{|l|}{ Outside cannulation } \\
\hline INTEGRIS team & $67(36 \%)$ \\
\hline Other facility team & $19(10 \%)$ \\
\hline Distance traveled (miles) & $56 \pm 54[1-198]$ \\
\hline Thoratec/Abbott Centrimag & 103 \\
\hline Maquet Cardiohelp & 73 \\
\hline \multicolumn{2}{|c|}{$\begin{array}{l}\text { ECMO, extracorporeal membrane oxygenation; BSA, body } \\
\text { surface area; MAP, mean arterial blood pressure; IABP, intra } \\
\text { aortic balloon pump; CRRT, continuous renal replacement } \\
\text { therapy; SOFA, Sequential Organ Failure Assessment; WBC, } \\
\text { white blood cell; BUN, blood urea nitrogen; AST, aspartate } \\
\text { aminotransferase. }\end{array}$} \\
\hline
\end{tabular}

edema/relevant LV distension in $4.9 \%(\mathrm{n}=9)$, lower limb ischemia in $4.3 \%(\mathrm{n}=8)$, HIT in $3.3 \%$ [ $\mathrm{n}=6$; LV/LA clot in $1.6 \%(\mathrm{n}=3)$, and infection (cannula-related, sepsis, VAP in $49.5 \%(n=91)]$. CRRT was necessary in $16.3 \%(n=30)$. A summary of all patient complications can be found in Table 4 .

\section{Outcomes analysis}

Overall survival was defined as survival to discharge from 


\begin{tabular}{|c|c|c|}
\hline Adverse event/complication $(n=184)$ & INTEGRIS Baptist rates, N (\%) & Literature-reported rates (\%) (Ref) \\
\hline $\begin{array}{l}\text { Bleeding } \\
\text { (systemic, retroperitoneal hematoma, tamponade) }\end{array}$ & $41(22.3)$ & $29.3-50(6-8)$ \\
\hline Cannula-related & $11(6.0)$ & $9.3(9)$ \\
\hline Gl bleed & $14(7.6)$ & $7(6)$ \\
\hline Oxygenator failure & $6(3.3)$ & $12.8(9)$ \\
\hline Oxygenator clots & $8(4.3)$ & Specific number not reported \\
\hline Hemolysis & $17(9.2)$ & $18(6)$ \\
\hline Seizure & $1(0.5) / 0.008$ EPPD & $1.8(10)$ \\
\hline Brain death & $4(2.2)$ & $7.9(10)$ \\
\hline Neurologic injury, non-ECMO related & $12(6.5)$ & Specific number not reported \\
\hline Pulmonary edema/LV distention & $9(4.9)$ & $12-68(12,13)$ \\
\hline Lower limb ischemia & $8(4.3)$ & $10-70(14)$ \\
\hline HIT & $6(3.2)$ & $8.3(15)$ \\
\hline LV/LA clot & $3(1.6)$ & Specific number not reported \\
\hline
\end{tabular}

ECMO, extracorporeal membrane oxygenation; GI, gastrointestinal; LV, left ventricle; HIT, heparin-induced thrombocytopenia; LA, left atrial; VAP, ventilator-associated pneumonia; CRRT, continuous renal replacement therapy; EPPD, event per patient day.

the hospital and was $39 \%$. A total of 83 patients (45\%) died from multisystem organ failure. The mean duration of ECMO support was $7.8 \pm 7.9(0.5-53)$ days with a median of 6 days. Total patient days were 1,430. Among these, $95(51.6 \%)$ patients were able to be weaned for recovery and $12(6.5 \%)$ went on to receive a durable MCS device.

\section{Discussion}

Despite the ease and applicability of VA-ECMO in patients requiring temporary MCS for $\mathrm{rCS}$, there continues to be significant complications associated with this advanced therapy (6). Although several smaller studies and anecdotal reports have described ECMO complication rates, the exact rates and specifics are not well understood, mainly in part due to differences in practice, as well as addressing overall experience and competence of an ECMO team. It can be difficult to determine whether overall complication rates are related to the severity of the disease state inherent in patients requiring VA-ECMO, or whether it is related to the VA-ECMO therapy itself. Of the multiple potential complications typically associated with VA-ECMO, the most commonly occurring can be classified as either device related, or patient related (19) (Table 1). 


\section{MDT}

rCS patients represent a high-acuity population, requiring complex and comprehensive care. Understandably, this cohort of patients would inevitably carry a high risk for complications. To address this concern, a MDT is ideal to continually manage the multiple needs of the acutely-ill; each member is able to offer their own individual expertise for the combined effort of complete, comprehensive care. The team may include an interventional cardiologist, a heart failure cardiologist, a cardiothoracic surgeon, an intensivist, an anesthesiologist, and advanced practice clinicians (including nurse practitioners and physician assistants, ICU nurses, and other clinical support staff) (1-3). The MDT is crucial in determining appropriateness of type and timing of interventions. MDT-based patient management requires a primary leader, who should be a qualified cardiovascular intensivist with training in heart failure, CT surgery or critical care. This individual's key roles include deciphering appropriateness of patient referral, triaging of patients based on acuity, rapidly assessing end-organ function based on laboratory and diagnostic testing, and determining proper device strategy for the clinical scenario of the patient $(2,3)$.

This study focused on the primary complications that VA-ECMO patients experienced through the course of support, and how the use of our own clinical practice guidelines and the oversight of a MDT were able to keep complication rates lower than reported incidence in a sicker cohort of patients (Table 3). In this analysis we started to report some complication rates as EPPD, as many of the complications are time related. Assigning an incidence rate can be misleading, as not every patient will be at risk of developing an ECMO-related complication during the same amount of time. Thus, reporting complication rates as EPPD help to establish a denominator, which is the measure of time each patient spends at-risk and allows for equal comparison. To our knowledge, there is no other literature which has reported complications as EPPD.

It is essential to recognize the importance of the ECMO circuit and its impact on helping to minimize adverse events. In our institution, we utilize a new-generation ECMO circuit, consisting of a magnetically levitated, centrifugal pump combined with a low pressure-drop oxygenator using Carmeda-coated (Medtronic, Minneapolis, MN, USA) tubing without bridges or connectors, helping to limit the formation of clots and minimize shear stress. Shorter circuits reduce the amount of biological contact between an artificial material and blood, thus the degree of inflammation is lessened. Newer ECMO technology allows for easier patient transport and bedside, ICU nursing oversight without the need for a $24 / 7$ perfusionist (20).

\section{VA-ECMO survival}

The Extracorporeal Life Support Organization (ELSO) Registry is a comprehensive database collecting data on multiple aspects of ECMO therapy, including 46 different ECMO complications in adult and pediatric patients. The most recent report describes overall ECMO use, with the number of centers utilizing ECMO increasing. Adults receiving ECMO for cardiac support from 1989-2015 show $41 \%$ survival to hospital discharge, with survival only increasing to $42 \%$ in the year 2015 (21). In our analysis, we report a $39 \%$ survival to hospital discharge, which includes patients where VA-ECMO was applied during or immediately after in-house cardiac arrest, and those who were transferred from an outside hospital where VA-ECMO was initiated.

\section{Bleeding, thromboembolic and coagulation complications}

As in all types of MCS, there is a delicate balance between bleeding and clotting. The challenge remains how to best maintain this balance in an already acutely-ill patient with clotting derangements and systemic anticoagulation requirements. Bleeding can occur both systemically or locally, particularly at the site of peripheral cannulation, if used. Although considered non-life-threatening, it can be a nuisance, requiring frequent dressing changes and blood product administration if copious. Systemic bleeding, such as in the GI tract, intrapericardial, retroperitoneal and intracranial, can have serious consequences, being difficult to manage in the setting of an already-compromised patient. The use of a Bleeding Practice Guideline (supplementary) at our institution has helped to standardize the treatment(s) utilized based on the etiology and location of bleeding. It also addresses scenarios with low pump flows and severe bleeding issues.

The physiology of coagulopathy is complex, multifactorial and impacted by systemic anticoagulation, loss of coagulation factors, thrombocytopenia, and hemolysis (see Figures 1 and 2: pump replaced due to catastrophic clotting). Other disorders such as heparin induced thrombocytopenia (HIT) and acquired von Willebrand syndrome (AvWS) may occur (9). Multiple studies have shown that patients treated 


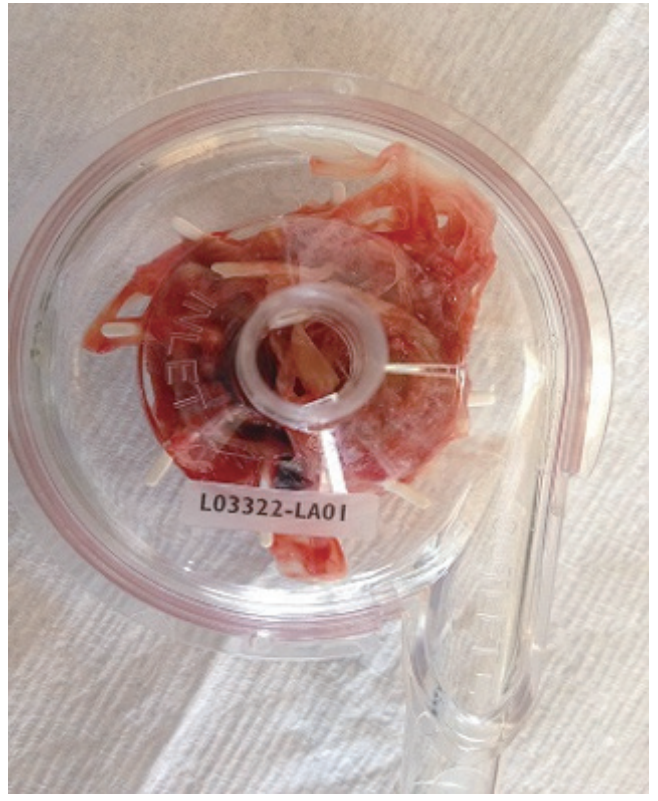

Figure 1 Oxygenator clot formation 1.

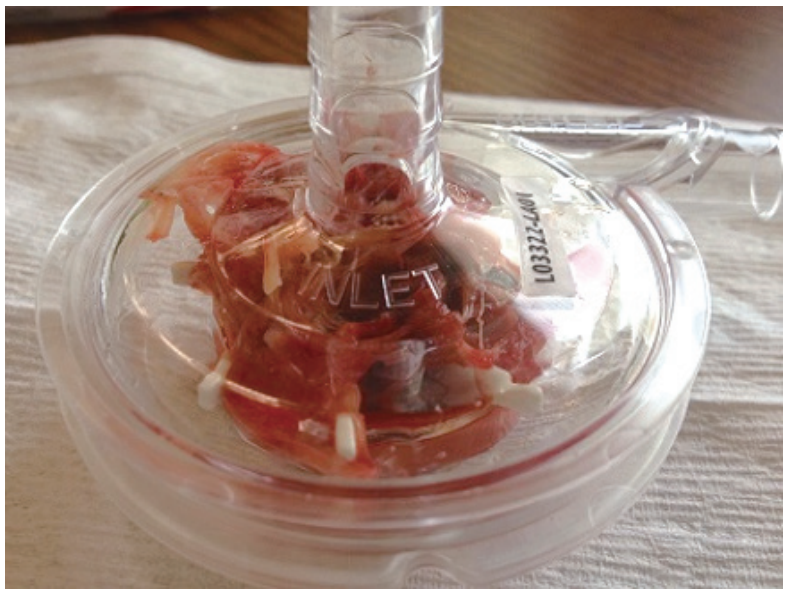

Figure 2 Oxygenator clot formation 2.

with MCS develop an AvWS, with the loss of high molecular weight von Willebrand factor (vWF) multimers (22). For patients who develop HIT, the use of direct thrombin inhibitors such as bivalirudin or argatroban can be used, however, there is limited data on the safety and efficacy of their use $(7,8)$.

There is a dearth of standardized coagulation approaches and monitoring methods for VA-ECMO-supported patients. The most commonly used systemic anticoagulation agent is unfractionated heparin. Direct thrombin inhibitors have been used but to a much lesser extent. Antiplatelet agents such as aspirin and dipyridamole are not commonly used. Anticoagulation monitoring varies at sites and includes ACT, aPTT, and anti-Xa levels. Thromboelastogram (TEG) is sometimes used to evaluate platelet function, however, not all centers routinely use this test $(20,23,24)$.

\section{Vascular complications and lower extremity ischemia}

With peripheral, percutaneous femoral arterial cannulation, vascular complications can be a risk, and lower extremity ischemia represents one of the more serious complications, with reported occurrence rates of $10-70 \%$ in adult VAECMO patients. Large-bore cannulas are inserted in the common femoral artery and can compromise blood flow to the lower extremity in a limb that is already most likely hypoperfused (14). This impeded circulation can cause lower limb ischemia and compartment syndrome, and if left untreated, can ultimately lead to amputation. Patients with smaller femoral vessels, and those requiring highdose vasopressors are at risk for compromised circulation. Careful oversight should be given to the insertion of the cannula with confirmation of placement by contrast X-ray of the extremity, to ensure that the cannula tip does not reside in the profunda $(14,25)$. Imaging also confirms that the cannula is not malpositioned. In our VA-ECMO population, bedside cannulation was performed in $>85 \%$ patients without bedside imaging.

Some centers have reported experience with the effective use of a noninvasive somatic oximeter (INVOS ${ }^{\mathrm{TM}}$ Cerebral/ Somatic Oximetry Adult Sensors, Medtronic, Minneapolis, MN, USA) to monitor the amount of oxygenated blood circulating to the lower extremities. This technology can help to identify changes in peripheral circulation, allowing for immediate intervention. Another measure to reduce limb ischemia is the use of an antegrade perfusion catheter when percutaneously cannulating for VA-ECMO. The placement of a distal perfusion catheter is performed on the ipsilateral superficial femoral artery with either a 5 or 7 French catheter depending on the patient's size. Arterial blood flow is then accomplished by connecting the distal perfusion catheter to the side port of the femoral artery cannula via a stopcock (26-28). At our center, attempts are made to place a distal perfusion catheter in all VAECMO patients. Some centers will initiate VA-ECMO via cannulation of the subclavian or axillary artery to reduce the risk of lower limb ischemia, however, this application has its own set of inherent risks, including upper extremity edema 
and inability to achieve high flow rates.

\section{Neurologic complications}

Neurologic injury represents a major complication during VA-ECMO therapy, often with devastating outcomes. Complications include cerebrovascular accidents (CVAs), including ischemic or hemorrhagic stroke, seizure, infarction, and brain death. Cerebral bleeding, thrombus, or air emboli can contribute to the development of a CVA and should be managed proactively to minimize its devastating impact.

Cerebral hypoperfusion or ischemia can occur as a consequence of desaturated blood within the aorta caused by mixing of oxygenated and deoxygenated blood, or watershed. This phenomenon, known as 'Harlequin's syndrome', is characterized by upper body, myocardial and cerebral ischemia. Mechanical ventilation should be maximized to ensure optimal oxygen levels and positive end-expiratory pressure (PEEP). Monitoring pulse oximetry and arterial blood gases via the right radial artery allows for ongoing evaluation of cerebral oxygenation $(10,15,29)$.

\section{Infection and significant inflammation}

Infection is one of the most common complications of VA-ECMO and occurs either systemically or locally (i.e., at the cannula insertion site). Reports have documented infection rates as high as $15-20 \%$, however, infection must be quantified based on etiology and timing. Higher yet, are the rates of nosocomial infections, with published incidence rates ranging from $8-64 \%$. These account for blood stream or peripherally-inserted cannula infections, and ventilator associated pneumonia (VAP) (16-18,30). It is understandable that critically-ill patients with multiple invasive lines, ongoing mechanical ventilation, VA-ECMO circuit exposure to blood, and higher length of ICU stay, are at higher risk for developing an infection. Some published strategies to help with early identification of impending nosocomial infections have incorporated (I) the routine use of surveillance cultures to help with early diagnosis; and (II) empiric antibiotic use, however, the risk for developing multi-drug resistant organisms and length and duration of antibiotics, must be considered prior to initiating antibiotic prophylaxis.

Studies have shown that the most common bloodstream pathogens identified in VA-ECMO-supported patients are
Staphylococcus aureus and other staphylococcus organisms, accounting for up to $38 \%$ of all bacteremia cases (16-18). Just as in non-ECMO patients, VAP is a risk for patients supported on mechanical ventilation. Patients on VAECMO are at risk for developing VAP, due to prolonged mechanical ventilation and lengthy immobility periods (30). Candida has been cited as one of the primary causes of pneumonia and blood stream infections, associated with a $47 \%$ mortality rate $(16,17)$. Also of note, up to $15 \%$ of blood stream infections in VA-ECMO patients are caused by fungal infections, with candida and aspergillus identified as primary culprit organisms, however, these rates tend to be similar to those of a non-ECMO supported, criticallyill patient residing in the ICU (31). The risk of sepsis progressing to multisystem organ failure is a major concern, which justifies the present clinical practice for early initiation of antibiotic therapy.

\section{Hemodynamic and LV dilation complications}

One of the biggest concerns with VA-ECMO therapy is the effect of retrograde flow in the aorta towards the LV. In some patients, this phenomenon causes increased LV afterload and ultimately, worsening LV function. Eventually, the LV dilates, myocardial blood flow decreases, left atrial (LA) pressures rise, and pulmonary edema ensues. LV distention has not been well documented or reported, primarily due to a lack of a consensus definition. Depressed LV contractility combined with increased $\mathrm{LV}$ afterload causes atrioventricular valve (AV) opening impairment, which could cause blood stagnation, increasing the risk for developing thrombus, and ultimately, CVA or AMI. Monitoring and achieving clinically acceptable LV unloading in VA-ECMO patients is essential.

The use of a hemodynamic catheter is crucial when evaluating volume status and filling pressures, with the overall goal of reducing volume overload, which can sometimes be achieved non-invasively, using vasodilator or inotropic agents, high-dose diuretics or hemofiltration. Large diameter ECMO cannulas should be considered if placing peripherally, however, this can be self-limiting for this type of approach, and central cannulation may be a better option if needed. If conservative methods are not sufficient, other methods to assist with decreasing blood volume should be considered, including the placement of an LV or LA venting catheter, atrial septostomy, trans-aortic catheter venting, venting via pulmonary artery drainage, and indirect $L V$ venting via IABP, 
TandemHeart, or Impella $(12,13,19,32)$.

\section{Cannulation-related complications}

Cannulation-related complications present a challenge when managing patients supported on VA-ECMO, with reported incidence rates up to $30 \%$. Complications can vary depending on the cannulation approach used, with bleeding occurring most frequently, in femoral, central, and axillary techniques. Pseudoaneurysm has also been reported, primarily after removal of femoral peripheral cannulas (19). This may necessitate the need for surgical repair if extensive. Limb ischemia and vascular complications also comprise a substantial number of cannula-related complications in this patient cohort. As mentioned above, measures to help to mitigate these types of complications include the use of a distal perfusion catheter, close monitoring of systemic anticoagulation, and careful selection of catheter size with assurance of securement of the cannula to prevent movement and migration. Hypoperfusion to the extremity could require fasciotomy or amputation, if left untreated. Interestingly, multiple studies have shown that patients who experienced a cannula-related complication showed no increase in mortality and were able to be successfully discharged (33).

VA-ECMO therapy continues to have concerning mortality rates. Mortality prediction models have been developed to help predict the risk of mortality in these acutely ill patients. The SAVE, ENCOURAGE, and SOFA scores have been used to predict overall patient survival and prognosis in those being supported with VA-ECMO. More contemporary scores have been developed to help to define disease severity and mortality risks such as the Simple Score, the EuroSCORE, and the CardShock Risk Score $(9,34)$.

\section{Limitations}

This is a retrospective analysis at a single center. Due to ethical concerns, there was no randomized prospective study and no control group other than the literature or ELSO data for complication comparisons. Medical approaches and modalities implemented prior to VA-ECMO initiation were not evaluated (e.g., timing of inotrope and vasopressor initiation and dosing, use of intravenous steroids, methylene blue, and use of short-term MCS). There was no outpatient follow-up with standardized evaluation of mental and/or physical status.

\section{Conclusions}

Complication and mortality rates remain a concern for the use and application of VA-ECMO for rCS patients. Future studies evaluating the use of best-practice guidelines, an experienced MDT and the impact they have on complication and mortality rates are necessary to further the field of short-term MCS and improve overall patient outcomes.

\section{Acknowledgements}

The authors thank Kimberly L. Eleuteri for her support.

\section{Footnote}

Conflicts of Interest: A El Banayosy, MD, D Horstmanshof, $\mathrm{MD}$, and JW Long, MD, PhD are consultants. The other authors have no conflicts of interest to declare.

\section{References}

1. Ergle K, Parto P, Krim SR. Percutaneous Ventricular Assist Devices: A Novel Approach in the Management of Patients with Acute Cardiogenic Shock. Ochsner J 2016;16:243-9.

2. Mebazaa A, Tolppanen H, Mueller C, et al. Acute heart failure and cardiogenic shock:a multidisciplinary practical guidance. Intensive Care Med 2016;42:147-63.

3. Doll JA, Ohman EM, Patel MR, et al. Clinical Decision Making:A Team - Based Approach to Patients in Cardiogenic Shock. Catheter Cardiovasc Interv 2016;88:424-33.

4. Thiele H, Ohman EM, Desch S, et al. Management of cardiogenic shock. Eur Heart J 2015;36:1223-30.

5. Takayama H, Takeda K, Doshi D, et al. Short-term continuous - flow ventricular assist devices. Curr Opin Cardiol 2014;29:266-74.

6. Zangrillo A, Landoni G, Biondi-Zoccai G, et al. A metaanalysis of complications and mortality of extracorporeal membrane oxygenation. Crit Care Resusc 2013;15:172-8.

7. Wang L, Wang H, and Hou X. Clinical Outcomes of Adult Patients Who Receive Extracorporeal Membrane Oxygenation for Postcardiotomy Cardiogenic Shock: A Systematic Review and Meta-Analysis. J Cardiothorac Vasc Anesth 2018;32:2087-93.

8. Petricevic M, Milicic D, Boban M, et al. Bleeding and 
Thrombotic Events in Patients Undergoing Mechanical Circulatory Support: A Review of Literature. Thorac Cardiovasc Surg 2015;63:636-46.

9. Harjola VP, Lassus J, Sionis A, et al. Clinical picture and risk prediction of short-term mortality in cardiogenic shock. Eur J Heart Fail 2015;17:501-9.

10. Lorusso R, Barili F, Di Mauro M, et al. In-hospital neurologic complications in adult patients undergoing venoarterial extracorporeal membrane oxygenation: results from the extracorporeal life support organization registry. Crit Care Med 2016;44:e964-72.

11. Xie A, Lo P, Yan TD, et al. Neurologic Complications of Extracorporeal Membrane Oxygenation: A Review. J Cardiothorac Vasc Anesth 2017;31:1836-46.

12. Soleimani B, Pae WE. Management of left ventricular distension during peripheral extracorporeal membrane oxygenation for cardiogenic shock. Perfusion 2012;27:326-31.

13. Truby LK, Takeda K, Mauro C, et al. Incidence and Implications of Left Ventricular Distention During Venoarterial Extracorporeal Membrane Oxygenation Support. ASAIO J 2017;63:257-65.

14. Ranney DN, Benrashid EB, Meza JM, et al. Vascular Complications and Use of a Distal Perfusion Cannula in Femorally Cannulated Patients on Extracorporeal Membrane Oxygenation. ASAIO J 2018;64:328-33.

15. Sokolovic M, Pratt AK, Vukicevic V, et al. Platelet Count Trends and Prevalence of Heparin-Induced Thrombocytopenia in a Cohort of Extracorporeal Membrane Oxygenator Patients. Crit Care Med 2016;44:e1031-7.

16. Haneke F, Schildhauer TA, Schlebes AD, et al. Infections and Extracorporeal Membrane Oxygenation: Incidence, Therapy, and Outcome. ASAIO J 2016;62:80-6.

17. Bréchot N, Luyt CE, Schmidt M, et al. Venoarterial Extracorporeal Membrane Oxygenation Support for Refractory Cardiovascular Dysfunction During Severe Bacterial Septic Shock. Crit Care Med 2013;41:1616-26.

18. Allou N, Pinto HL, Persichini R, et al. Cannula-Related Infection in Patients Supported by Peripheral ECMO: Clinical Microbiological Characteristics. ASAIO J 2018. [Epub ahead of print].

19. Wong JK, Melvin AL, Joshi DJ, et al. Cannulation-Related complications on Veno-Arterial Extracorporeal Membrane Oxygenation: Prevalence and Effect on Mortality. Artif Organs 2017;41:827-34.

20. Ghodsizad A, Koerner MM, Brehm CE, et al. The role of extracorporeal membrane oxygenation circulatory support in the 'crash and burn' patient:from implantation to weaning. Curr Opin Cardiol 2014;29:275-80.

21. Sun T, Guy A, Sidhu A, et al. Veno-arterial extracorporeal membrane oxygenation (VA-ECMO) for emergency cardiac support. J Crit Care 2018;44:31-8.

22. Malfertheiner MV, Pimenta LP, von Bahr V, et al. Acquired von Willebrand syndrome in respiratory extracorporeal life support: a systematic review of the literature. Crit Care Resusc 2017;19:45-52.

23. Baumann Kreuziger L, Massicotte MP. Mechanical circulatory support: balancing bleeding and clotting in high-risk patients. Hematology Am Soc Hematol Educ Program 2015;2015:61-8.

24. Ghodsizad A, Koerner MM, El-Banayosy A, et al. Extracorporeal Membrane Oxygenation (ECMO): An Option for Cardiac Recovery from Advanced Cardiogenic Shock. Heart Surg Forum 2017;20:E274-7.

25. Mohite PN, Fatullayev J, Maunz O, et al. Distal Limb Perfusion: Achilles' Heel in Peripheral Venoarterial Extracorporeal Membrane Oxygenation. Artif Organs 2014;38:940-4.

26. Aziz F, Brehm CE, El-Banyosy A, et al. Arterial complications in patients undergoing extracorporeal membrane oxygenation via femoral cannulation. Ann Vasc Surg 2014;28:178-83.

27. Mandawat A, Rao SV. Percutaneous Mechanical Circulatory Support Devices in Cardiogenic Shock. Circ Cardiovasc Interv 2017;10(5).

28. Banfi C, Pozzi M, Brunner ME, et al. Veno - arterial extracorporeal membrane oxygenation; an overview of different cannulation techniques. J Thorac Dis 2016;8:E875-85.

29. Prasad A, Ghodsizad A, Brehm C, et al. Refractory Pulmonary Edema and Upper Body Hypoxemia During Veno-Arterial Extracorporeal Membrane Oxygenation-A Case for Atrial Septostomy. Artif Organs 2018;42:664-9.

30. Bouglé A, Bombled C, Margetis D, et al. Ventilatorassociated pneumonia in patients assisted by venoarterial extracorporeal membrane oxygenation support: Epidemiology and risk factors of treatment failure. PLoS One 2018;13:e0194976.

31. Cavayas YA, Usuff H, Porter R. Fungal infections in adult patients on extracorporeal life support. Critical Care 2018;22:98.

32. Meani P, Gelsomino S, Natour E, et al. Modalities and Effects of Left Ventricle Unloading on Extracorporeal Life support: a Review of the Current Literature. Eur J Heart Fail 2017;19:84-91. 
33. Rajagopal K. Left Ventricular Distension in Veno-arterial Extracorporeal Membrane Oxygenation: From Mechanics to Therapies. ASAIO J 2019;65:1-10.

34. Wang L, Wang H, Hou X. Clinical Outcomes of Adult

Cite this article as: Koerner MM, Harper MD, Gordon CK, Horstmanshof D, Long JW, Sasevich MJ, Neel JD, El Banayosy A. Adult cardiac veno-arterial extracorporeal life support (VAECMO): prevention and management of acute complications. Ann Cardiothorac Surg 2019;8(1):66-75. doi: 10.21037/ acs.2018.12.09
Patients Who Receive Extracorporeal Membrane Oxygenation for Postcardiotomy Cardiogenic Shock: A Systematic Review and Meta-Analysis. J Cardiothorac Vasc Anesth 2018;32:2087-93. 


\section{Anticoagulation protocol}

* Administer heparin 75-120 IU/kg bolus for extracorporeal membrane oxygenation (ECMO) cannulation;

* For patients that have received thrombolytic therapy or cardiac surgery, no Heparin initially;
* Continuous heparin infusion during ECMO therapy with PTT goal of 50-60 s;

* For ECMO weaning process (lower pump speed/flow $<2.5 \mathrm{~L} / \mathrm{min}$,) increase target PTT level to $60-80 \mathrm{~s}$;

* For heparin induced thrombocytopenia (HIT) positive patient, start argatroban;

* Stop heparin in case of severe bleeding. 